Ann. Biol. anim. Bioch. Biophys., I976, 16 (I), 97-100.

\title{
LES CHANGEMENTS
}

\section{DE LA CONCENTRATION EN MAGNÉSIUM PLASMATIQUE CHEZ UN GROUPE D'ANIMAUX APRES ÉTABLISSEMENT D'UN « BY PASS » JÉJUNO-ILÉAL}

\author{
F. ANNONI et F. LONGONI \\ Istituto di Patologia Chirurgica III, \\ Università di Milano, \\ Istituto di Chirurgica Plastica Ricostruttiva, \\ Milano (Italie)
}

\section{RÉSUMÉ}

La mise hors circuit de $90 \mathrm{p}$. Ioo environ de la surface absorbante du jéjunum et de l'iléon provoque peu de troubles de la magnésémie chez le Porc, dont le côlon est particulièrement développé. Par contre chez le Chien (beaucoup plus comparable à l'Homme à cet égard), on observe une hypomagnésémie importante, persistant un an après l'opération.

\section{INTRODUCTION}

Depuis une dizaine d'années diverses tentatives ont été faites pour traiter 1'obésité grave de 1'Homme en excluant chirurgicalement une partie plus ou moins importante de l'intestin grêle (" by pass " jéjuno-iléal).

Actuellement, cette intervention se pratique de préférence par anastomose termino-terminale entre le jéjunum et l'iléon, en conservant environ $30 \mathrm{~cm}$ de chacune de ces zones. La part d'intestin exclue est fermée à l'extrémité proximale par une suture en bourse et anastomosée au côlon à l'extrémité distale.

Cette opération entraîne au bout de deux années environ, un amaigrissement considérable des sujets lié à l'exclusion de la plupart de la surface absorbante intestinale.

La diminution de l'absorption porte non seulement sur les nutriments énergétiques, mais aussi sur tous les autres, y compris les minéraux.

Des observations d'hypomagnésémies à la suite de résections importantes de 
l'intestin grêle ont déjà été publiées (HEAToN et al., I967) avec des taux de Mg plasmatique diminuant jusqu'à 0,2 à $0,3 \mathrm{mEq} /$ /itre chez 1'Homme.

En ce qui concerne le "by-pass » jéjuno-iléal, SwEnson et al. (1974) ont observé cinq cas d'hypomagnésémie chez des malades opérés.

Cette opération étant désormais pratiquée à l'Institut de Pathologie chirurgicale III de 1'Université de Milan, il nous a paru nécessaire d'étudier sur des animaux d'expérience (porc et chien) son retentissement sur la magnésémie.

\section{MATÉRIEI, ET MÉTHODES}

Huit porcs nains, des deux sexes, ont été opérés selon des modalités analogues à celles de l'Homme, c'est-à-dire que l'on a conservé environ $30 \mathrm{~cm}$ de jéjunum et $30 \mathrm{~cm}$ d'iléon en réalisant une anastomose termino-terminale, et que la partie exclue a été suturée en bourse à l'extrémité proximale, et anastomosée au côlon à l'extrémité distale. Toutefois la longueur du côlon est beaucoup plus grande chez le Porc que chez l'Homme, ce qui explique que l'effet sur l'amaigrissement ait été plus faible chez nos animaux.

Deux groupes de six chiens Beagles ont été opérés, selon deux modalités différentes : le premier groupe ("by pass court") a subi un court-circuit tel que l'on a conservé seulement $\mathrm{I}_{5} \mathrm{~cm}$ de jéjunum et $15 \mathrm{~cm}$ d'iléon (30 cm en tout). Pour le second groupe ( by pass long ") on a conservé 30 à $35 \mathrm{~cm}$ de jéjunum et 20 à $25 \mathrm{~cm}$ d'iléon (50 à $60 \mathrm{~cm}$ en tout).

On peut considérer que ces ablations correspondent sensiblement à celles réalisées chez l'Homme obèse (" by pass court " avec des anses jéjunales et iléales de I $5 \mathrm{~cm}$, soit $30 \mathrm{~cm}$ en tout, et "by pass long" avec une anse jéjunale de $22 \mathrm{~cm}$ et une anse iléale de $48 \mathrm{~cm}$ ).

Un an après l'opération, 6 chiens sur. I 2 sont encore en vie. Trois sont morts spontanément, trois ont été sacrifiés pour étudier l'aspect histologique des tissus.

Des prises de sang ont été effectuées aux temps indiqués sur les figures, pendant 220 jours chez les porcs et un an chez les chiens.

Le magnésium sanguin a été dosé par spectrophotométrie d'absorption atomique.

\section{RÉSULTATS ET DISCUSSION}

Chez le Porc, la figure I montre que le magnésium plasmatique a été affecté assez faiblement, et pendant un temps assez court, le retour à la normale s'effectuant en 6 mois environ. Il semble que chez cet animal le développement anatomique impor-

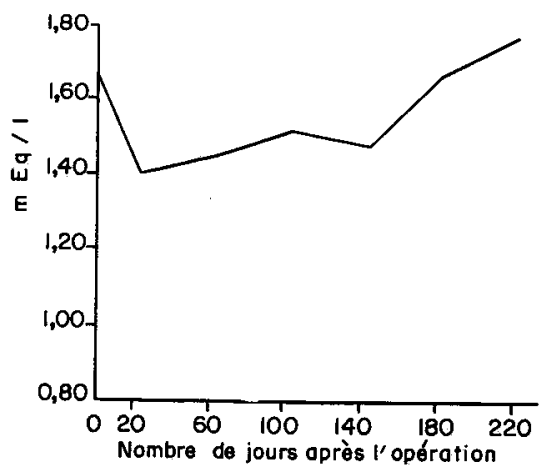

FIG. I. -- Evolution post-opératoire du magnésium plasmatique chẽ des porcs après réalisation d'un " by pass "jéjụno-iléal 
tant du côlon corresponde à une capacité d'absorption considérable, capable de compenser l'ablation d'une part importante de l'intestin grêle. Cela peut aussi s'expliquer par un besoin en magnésium beaucoup plus faible chez le Porc (500 p.p.m. dans l'aliment sec) que chez l'Homme (et peut-être chez le Chien).

Chez le Chien (fig. 2) la baisse de la magnésémie est beaucoup plus considérable, la diminution étant significativement plus forte dans le cas d'un " by pass court " pendant la période allant de 40 à $\mathbf{1 2 0}$ jours après l'opération.

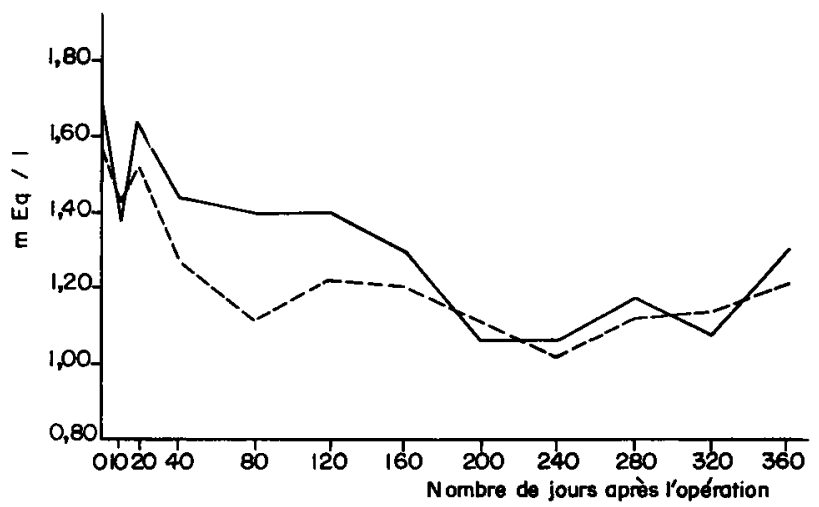

FIG. 2. - Évolution post-opératoire du magnésium plasmatique chez des chiens après réalisation d'un " by pass "jéjuno-iléal, long (_- ) ou court (--..--)

La baisse rapide et temporaire observée dans les ro premiers jours est probablement liée à un phénomène de stress, et aussi au jeûne presque complet imposé aux animaux dans la période post-opératoire.

Le minimum observé individuellement a été de $0,65 \mathrm{mEq} / 1 \mathrm{chez}$ un chien opéré d'un " by pass court », 80 jours après l'opération.

Un an après l'opération, malgré une légère tendance à la remontée des valeurs du $\mathrm{Mg}$ plasmatique, la moyenne reste aux environs de $1,25 \mathrm{mEq} / 1$.

L'examen histologique chez des animaux opérés depuis 240 jours a montré une hypertrophie des villosités intestinales du grêle, ainsi qu'un allongement de la partie restée en place. Ces observations sont à relier à la légère remontée de la magnésémie, ainsi qu'à l'amélioration sensible de l'état général des animaux à partir de ce moment.

\section{CONCLUSION}

Nous observons donc chez le Chien des modifications de la magnésémie comparables à ce qui a été décrit chez 1'Homme après réalisation d'un " by-pass " jéjunoiléal.

Il est vraisemblable que l'hypomagnésémie peut être attribuée à la malabsorption de $\mathrm{Mg}$, comme de tous les autres nutriments.

L'amélioration de la magnésémie après 240 jours correspond à la stabilisation du poids chez les opérés, et traduit sans doute la mise en jeu de certains phénomènes compensatoires (hypertrophie des villosités, allongement de la partie restée en place). 
Ainsi cette opération, qui exclut environ 90 p. Ioo de la surface absorbante du jéjunum et de l'iléon, induit des changements importants du bilan électrolytique du magnésium et rend nécessaire un contrôle post-opératoire sérieux de la magnésémie, aspect trop souvent négligé selon nous.

Reçu pour publication en juin 1975.

\title{
SUMMARY
}

\author{
CHANGES IN PLASMA MAGNESIUM LEVEL IN A GROUP \\ OF ANIMALS FOLLOWING CREATION OF A JEJUNO-ILEAL BY-PASS
}

About 90 p. Ioo of the absorbant surface of the jejunum and ileum in swine is disconnected. The plasma magnesium level is little upset because the colon is very developed. However, considerable hypomagnesia persists one year after the operation in dog, which is more similar to man in this respect.

\section{RÉFÉRENCES BIBLIOGRAPHIQUES}

Barbieri L. L., Vancini B., Civiero G., I96o. Studio sulla magnesiemia in alcune condizioni patologiche. Arch. Patol. Clin. Med., 37, 17*30.

CARD W. I., Marks I. N., I958. A case of magnesium deficiency. Ciba Found. Coll. on Ageing, 4, 30 I.

Fletcher R. F., Henly A. A., Sammong H. G., Squire J. R., ig6o. A case of magnesium deficiency following massive intestinal resection. Lancet, $\mathbf{1}, \mathbf{5 2 2 - 5 2 5}$.

FIINK E. B., I956. Magnesium deficiency syndrome in man. J. am. Med. Ass,, 160, I406-I409.

Flink E. B., McCollister R., Prasad A. S., Melgy J. C., Doe R. P., I95\%. Evidences for clinical magnesium deficiency. Ann. Int. Med., 47, 956-968.

Gerst P. H., Porter M. R., Frshman R. A., 1964. Symptomatic Mg deficiency in surgical patients. Ann. Surg., 159, 402-406.

Hammarsten J. F., Smith W. O., 1957. Symptomatic magnesium deficiency in man. New England J. Med., 256, 897-899.

Haynes B. W., Jr, Crawford E. S., De Bakey M. E., I952. Magnesium metabolism in surgical patients. Exploratory observations. Ann. Surg., 186, 659-667.

Heaton F. W., Clark C. G., Goligher J. C., r967. Magnesium deficiency complicating intestinal surgery. Brit. J. Surg., 54, 4I-45.

Heaton F. W., Fourman P., I965. Magnesium deficiency and hypocalcaemia in intestinal malabsorp tion. Lancet, 2, 50-52.

Henzel J. H., De Weese M. S., Ridenhour G., I967. Significance of Mg and Zn Metabolism in the Surgical Patient. Arch. Surg., 95, go.

LeVey S., Abbott W. E., Krieger H., Davis J. H., I956. Metabolic alteration in surgical patients. VIII. Studies involving iron and magnesium metabolism in patients with gastrointestinal drainage. J. Lab. Clin. Med., 47, 437-443.

Maсbетн R. A., Маввотт J. D., 1964. Mg Balance in the Postoperative patient. Surg. Gyn. Obst., $118,748-760$.

Mark J. B. D., Hayes M. A., I96r. Studies of calcium and magnesium metabolism during surgical convalescence. Surg. Gyn. Obst., 113, $213^{-2} \mathrm{I} 8$.

Sawyer R. B., Drew M. A., Gesink M. H., Sawyer K. C., Sawyer Jr. K. C., 1969. Postoperative Mg metabolism. Arch. Surg., 100, 343-348.

Sмiтн W. O., 1963. Mg Deficiency in the surgical patient. Am. J. Card., 13, 667-67o.

SWenson S. A., Lewis J. W., Sebry K. R., I974. Magnesium metabolism in man with special reference to jejunoileal bypass for obesity. Am. J. Surg., 127, 250-255.

Thoren L., 1962. Magnesium deficiency : studies in two cases of acute fulminant colitis treated by colectomy. Acta Chir. Scend., 124, 134-143.

Thoren L., I963: Magnesium deficiency in gastrointestinal fluid loss. Acta Chir. Scand., suppl. 306. 